\title{
Antimutagenic effects of ellagic acid, rutin and psoralen against aflatoxin B1
}

\section{Jolanta Pierzynowska ${ }^{1}$ and Elżbieta Grzesiuk ${ }^{2}$}

\author{
'Department of Human Nutrition, \\ Warsaw Agricultural University \\ Nowoursynowska 166, 02-787 Warszawa, Poland \\ 2Institute of Biochemistry and Biophysics, Polish Academy of Sciences \\ Pawinskiego 5A, 02-106 Warszawa, Poland
}

\begin{abstract}
Aflatoxin Bl (AFBI) is frequently found in food and feedstuffs. It is a potent mutagenic and carcinogenic (e.g. hepatocellular carcinoma) mycotoxin produced by Aspergillus flavus, A. parasiticus and A. nomius. At the cellular level, after appropriate metabolism of AFBI in cytochrome P450-dependent reactions, the resulting epoxide binds to DNA and induces point mutations. Using a Salmonella microsuspension assay we have examined: (1) mutagenic activity of AFB1 in the presence of the S9 fraction in TA98 and TA 100 tester strains, (2) antimutagenic activity of ellagic acid (EA), rutin (RU) and psoralen (PS) against AFBl. The result of our investigations, was that the TA98 strain was more sensitive than the TA 100 to mutagenic action of AFB1 and antimutagenic action of EA, RU and PS. Among the three compounds PS showed the highest anti-AFBI activity; 20 ng of PS inhibited the response to 10 ng of AFB1 by $90 \%$, while the same amount of EA inhibited AFBI by $82 \%$, and RU was less potent still (only $60 \%$ inhibition).
\end{abstract}

KEY WORDS: aflatoxin BI, antimutagenic substances

\section{INTRODUCTION}

Aflatoxin $\mathrm{Bl}$ (AFBl) is a potent environmental carcinogen produced by the common molds Aspergillus flavus, A. parasiticus and $A$. nomius. These fungi are frequently found in feeds such as maize, peanuts and cotton seeds (Hsieh, 1989; Carillo et al., 1990; Liu and Massey, 1992). Epidemiological studies have established that it is one of important risk factors for human and animal hepatocellular carcinoma. AFBl requires metabolic activation by the cytochrome P450 enzyme 
system to produce the AFB1-8,9-epoxide which is an absolute requirement for AFB1 to manifest its mutagenic, carcinogenic and DNA binding action (Gurtoo and Dave, 1975; Campbell and Hayes, 1976; Essigmann et al., 1982). Recently, a low incidence of cancer has been connected with consumption of fresh fruit and vegetables. In addition to vitamins $A, C$ and $\beta$-carotene (Ho, 1992; Kuo et al., 1992), many fruits and vegetables are also known to contain phenolic compounds. These naturally occurring compounds, particularly phenolic acids and polyhydroxylated flavonoids have been shown to possess anticarcinogenic activity toward a variety of chemicals (Wattenberg et al., 1970, 1980; Lesca, 1983; Stich and Powric, 1984; Elangova et al., 1994). Some of these compounds inhibit carcinogenity in animals and humans (Stavric, 1994) and some can modulate various key cellular enzyme function (Ho, 1992). Among these effective polyphenols are ellagic acid (EA) and rutin (RU). EA is a phenolic bislactone (flavonoid) which has been found in vegetables and fruits such as strawberries, blackcurrents, rasberries and walnuts (Lord et al., 1989; Josephy et al., 1990; Rossi et al., 1991). RU is a glycoside of flavonol quercetin, which is present in a wide variety of vegetables and fruits, especially in blueberries. A compound with similar chemical structure is psoralen (PS), a furocoumarin which is widespread in plants, mostly among members of Leguminosae, Umbelliferae and Rutaceae families (Bisagni, 1992). The aim of the present study was to investigate the effects of EA, RU and PS on the mutagenicity of AFB1 using the Salmonella liquid incubation assay - Ames test (Ames et al., 1975).

\section{MATERIAL AND METHODS}

\section{Chemicals}

Aflatoxin B1 (AFB1), ellagic acid (EA), rutin (RU), psoralen (PS) and dimethyl sulfoxide (DMSO) were obtained from Sigma Chemicals. Stock solutions of AFB1, EA, RU and PS were prepared in DMSO; at a concentration of $2 \mathrm{mg} / \mathrm{ml}$ in the case of AFB1, and $4 \mathrm{mg} / \mathrm{ml}$ for EA, RU and PS.

\section{Salmonella mutagenicity test}

The Ames test with further modifications (Kado et al., 1983) was used to rest for mutagenicity. Tester strains TA98 and TA100 were obtained from the Department of Biochemistry, Medical School, Warsaw (Poland). The S9 fraction was prepared from livers of rats treated with Aroclor 1254 according to the procedure of Ames (1975). Bacteria were grown overnight in LB medium containing ampicillin $(50 \mathrm{mg} / \mathrm{ml})$ to approx. $1-2 \times 10^{9}$ cells $/ \mathrm{ml}$, harvested by centrifugation and 
resuspended in phosphate buffer $\mathrm{pH} 7.4$ to a final concentration of $1-2 \times 10^{10} \mathrm{cells} / \mathrm{ml}$. $0.1 \mathrm{ml}$ of the bacteria was put into $12 \times 75 \mathrm{~mm}$ sterile plastic tube. Apart from the bacteria, each tube contained $0.1 \mathrm{ml}$ of S9 mix $(8 \mathrm{mM} \mathrm{MgCl} ; 33 \mathrm{mM} \mathrm{KCl} ; 100$ $\mathrm{mM}$ sodium phosphate $\mathrm{pH} 7.4 ; 5 \mathrm{mM}$ glucose-6-phosphate; $4 \mathrm{mM}$ NADP and 40 $\mathrm{ml} / \mathrm{ml}$ of S9 fraction as well as respectively: AFB1, EA, RU or PS). Antimutators were added at the same time as AFB1. During addition of all ingredients the tubes were kept on ice but afterwards they were incubated at $37^{\circ} \mathrm{C}$ in the dark with rapid shaking. After $90 \mathrm{~min}$, a $2 \mathrm{ml}$ melted top agar containing $90 \mathrm{nmol}$ of histidine and biotin (Ames et al., 1975) was added into each tube. Combined solutions were poured onto minimal glucose plates and incubated at $37^{\circ} \mathrm{C}$. The colonies of His + mutants were counted after $48 \mathrm{~h}$ incubation.

\section{RESULTS}

AFB1 caused mutagenicity of both the TA98 and TA100 strains of Salmonella typhimurium, with linear dose-response curves as illustrated in Figure 1. Tester strain TA98 was about $60 \%$ more sensitive to the mutagenic action of AFB1 than

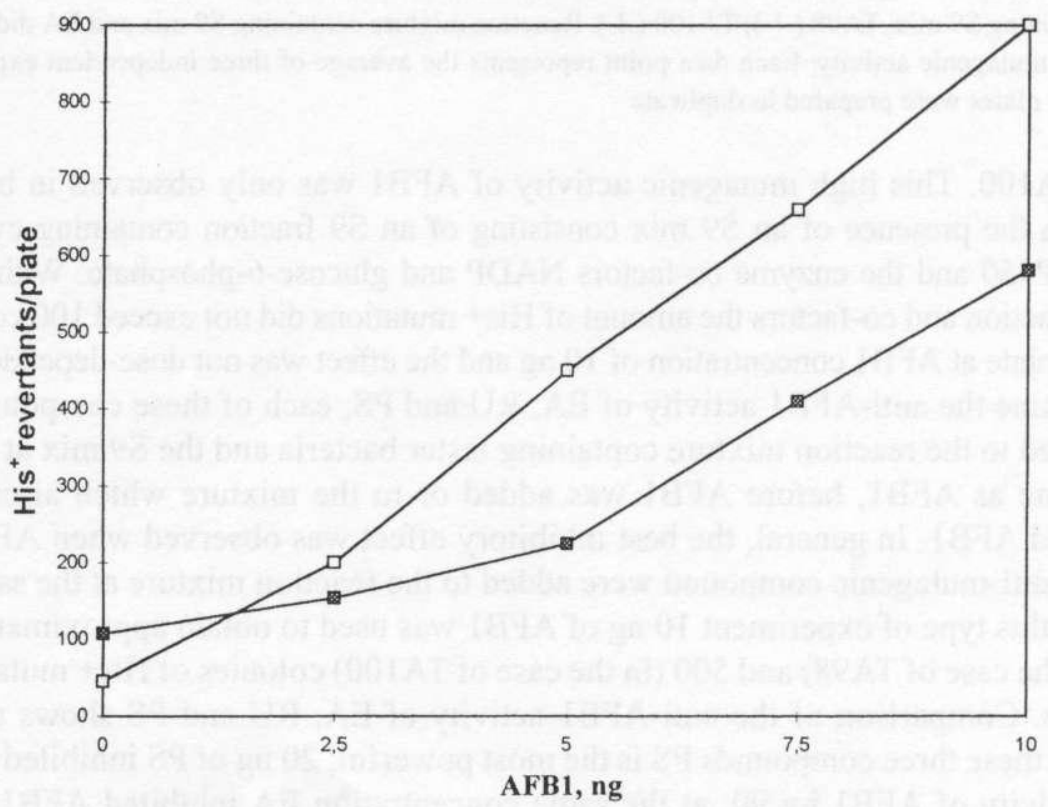

Figure 1. Dose-response curves of AFB1 mutagenicity in the presence of S9 mix in TA98 (-) and TA100 (-n-) tester strains. Spontaneous His+ mutation level was 40110 colonies/plate and 240150 colonies/plate for TA98 and TA100, respectively. Each data point represents the average of three independent experiments, the plates were prepared in duplicate 


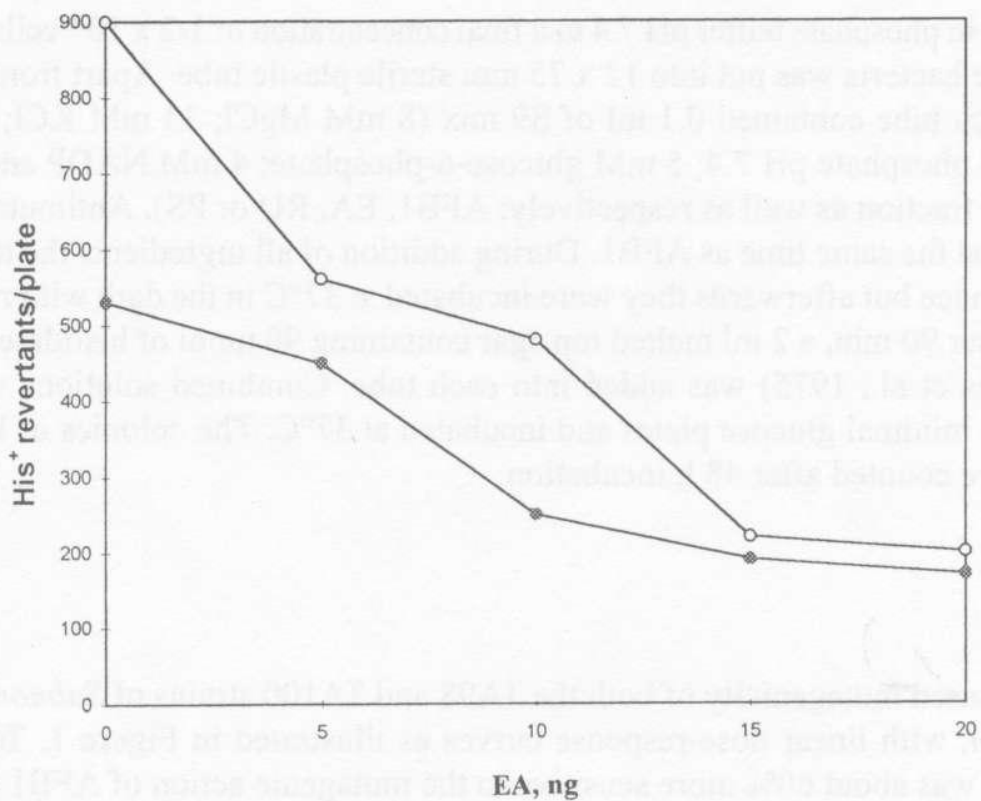

Figure 2. Anti-AFB1 action of EA. 10ng of AFB1 and different amounts of EA were added into each tube containing S9 mix. TA98 (-!-); TA100 (-1-). Reaction mixture containing S9 mix and EA did not show any mutagenic activity. Each data point represents the average of three independent experiments, the plates were prepared in duplicate

strain TA100. This high mutagenic activity of AFB1 was only observed in both strains in the presence of an S9 mix consisting of an S9 fraction containing cytochrome P450 and the enzyme co-factors NADP and glucose-6-phosphate. Without the $\mathrm{S} 9$ fraction and co-factors the amount of His+ mutations did not exceed 100 colonies per plate at $\mathrm{AFB} 1$ concentration of $10 \mathrm{ng}$ and the effect was not dose-dependent.

Examine the anti-AFB1 activity of EA, RU and PS, each of these compounds was added to the reaction mixture containing tester bacteria and the S9 mix at the same time as AFB1, before AFB1 was added or to the mixture which already contained AFB1. In general, the best inhibitory effect was observed when AFB1 and the anti-mutagenic compound were added to the reaction mixture at the same time. In this type of experiment $10 \mathrm{ng}$ of AFB1 was used to obtain approximately 900 (in the case of TA98) and 500 (in the case of TA100) colonies of His+ mutants per plate. Comparison of the anti-AFB1 activity of EA, RU and PS shows that between these three compounds PS is the most powerful; $20 \mathrm{ng}$ of PS inhibited the mutagenicity of AFB1 by 90; at the same concentration EA inhibited AFB1 by $82 \%$; RU was less active and gave only $60 \%$ of AFB1 inhibition. Strain TA98 responded better than TA100 to the anti-AFB1 action of EA and PS. For instance, at the concentration of $20 \mathrm{ng}$ EA inhibited AFB1 by $82 \%$ in strain TA98 and by 
PIERZYNOWSKA J., GRZESIUK E.

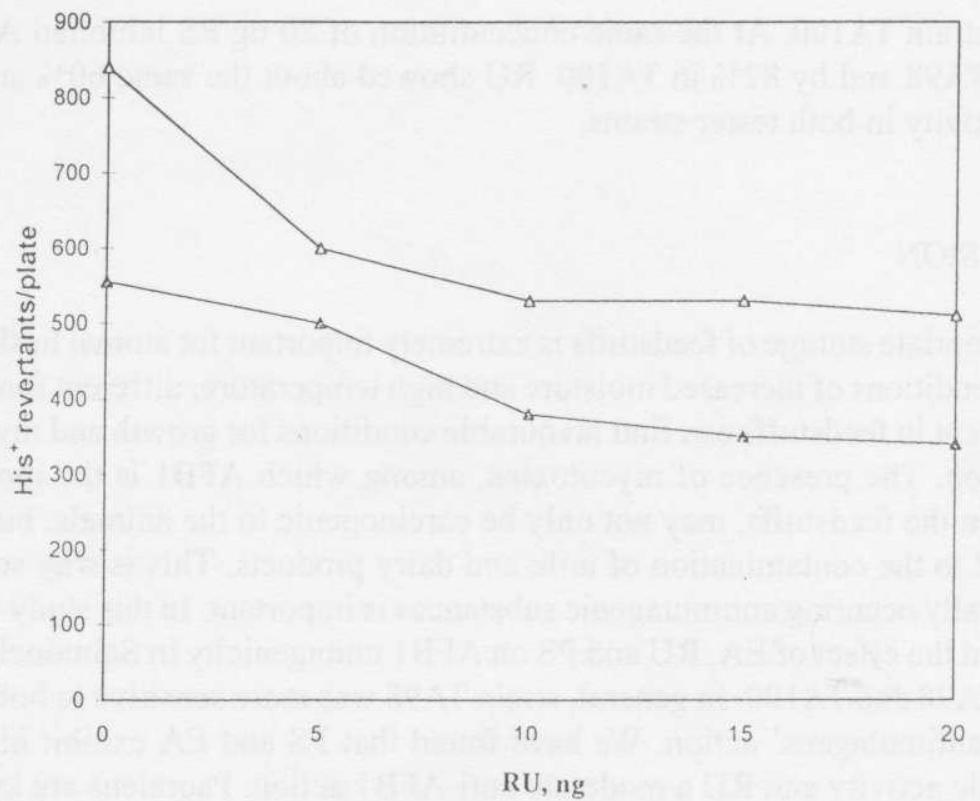

Figure 3. Anti-AFB1 action of RU. TA98 (-D-); TA100 (-D-). For details see Figure 2

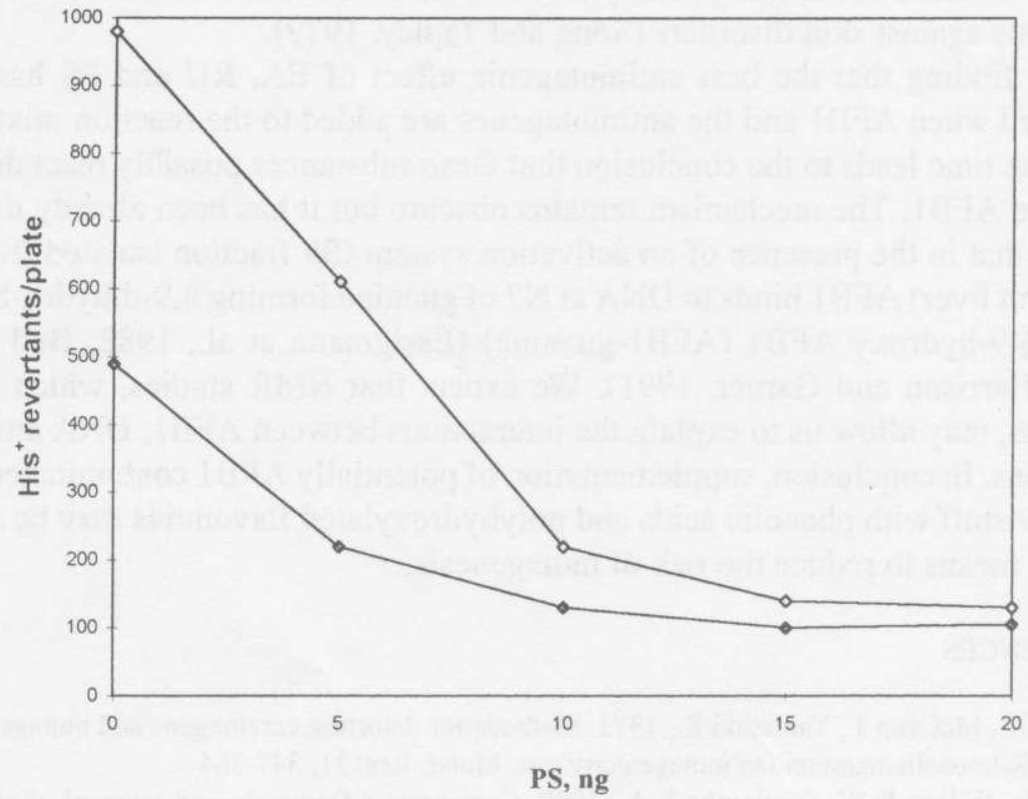

Figure 4. Anti-AFB1 action of PS. TA98 (- - ); TA100 (-t-). For details see Figure 2 
$76 \%$ in strain TA100. At the same concentration of 20 ng PS inhibited AFB1 by $90 \%$ in TA98 and by $82 \%$ in TA100. RU showed about the same $60 \%$ antimutagenic activity in both tester strains.

\section{DISCUSSION}

Appropriate storage of feedstuffs is extremely important for animal husbandary. Under conditions of increased moisture and high temperature, different fungal species present in feedstuffs can find favourable conditions for growth and mycotoxin production. The presence of mycotoxins, among which AFB1 is the most often present in the feedstuffs, may not only be carcinogenic to the animals, but it may also lead to the contamination of milk and dairy products. This is why searching for naturally occuring antimutagenic substances is important. In this study we have examined the effect of EA, RU and PS on AFB1 mutagenicity in Salmonella tester strains TA98 and TA100. In general, strain TA98 was more sensitive to both AFB1 and the antimutagens' action. We have found that PS and EA exhibit high antimutagenic activity and RU a moderate anti-AFB 1 action. Psoralens are known to possess photo-induced melanizing properties but we have shown, for the first time, a high anti-AFB 1 activity of one of them. There are several furocoumarins which have a similar structure but different residues. We plan to examine the anti-AFB1 activity of some of them, especially those which are known to be strong therapeutic agents against skin disorders (Song and Tapley, 1979).

The finding that the best antimutagenic effect of EA, RU and PS has been observed when AFBl and the antimutagenes are added to the reaction mixture at the same time leads to the conclusion that these substances possibly react directly with the AFB1. The mechanism remains obscure but it has been already demonstrated that in the presence of an activation system ( $\mathrm{S} 9$ fraction isolated from induced rat liver) AFB1 binds to DNA at N7 of guanine forming 8,9-dihydro-8-(N7guanyl)-9-hydroxy AFBl (AFBl-guanine) (Essigmann et al., 1982; Ball et al., 1990; Harrison and Garner, 1991). We expect that NMR studies, which are in progress, may allow us to explain the interactions between AFB1, DNA and antimutagens. In conclusion, supplementation of potentially AFBI contaminated food and fecdstuff with phenolic acids and polyhydroxylated flavonoids may be an important means to reduce the risk of mutagenesis.

\section{REFERENCES}

Ames B.N., McCann J., Yamasaki E., 1975. Methods for detecting carcinogens and mutagens with the Salmonella/mammalian mutagenicity test. Mutat. Res. 31, 347-364

Ball R.W., Wilson D.W., Coulombe R.A., 1990. Comparative formation and removal of aflatoxin Bl-DNA adducts in cultured mammalian tracheal epithelium. Cancer Res. 50, 4918-4922 
Bisagni E., 1992. Synthesis of psoralens and analogues. J.Photochem. B. Biol. 14, 23-46

Campbell T.C., Hayes J.R., 1976. The role of aflatoxin metabolism in its toxic lesion. Toxicol. Appl. Pharmacol. 35, 199-222

Carrillo M.C., Carnovale C.E., Monti J.A., 1990. Effect of aflatoxin B1 treatment in vivo on the in vitro activity of hepatic and extrahepatic glutathione S-transferase. Toxicol. Lett. 50, 107-116

Elangova V., Sekar N., Govindswamy S., 1994. Chemopreventive potential of dietary bioflavonoids against 20-methylcholanthrene-induced tumorigenesis. Cancer Lett. 87, 107-113

Essigmann J.M., Croy R.G., Bennett R.A., Wogan G.N., 1982. Metabolic activation of aflatoxin B1: Patterns of DNA adduct formation, removal, and excretion in relation to carcinogenesis. Drug Metab. Rev. 13, 581-602

Gurtoo H.L., Dave C.V., 1975. In vitro metabolic conversion of aflatoxins and benzo(a)pyrene to nucleic acid-binding metabolites. Cancer Res. 35, 382-389

Harrison J.C., Garner R.C., 1991. Immunological and HPLC detection of aflatoxin adducts in human tissues after an acute poisoning incident in S.E. Asia. Carcinogenesis 12, 741-743

Ho C., 1992. Phenolic compounds in food, In: C.Ho, C.Y.Lee, M. Huang (Editors). Phenolic compound in food and their effects on health I. Analysis, occurrence and chemistry. American Chemical Society, Washington DC, pp. 1-7

Hsieh D.P.H., 1989. Potential human helth hazards of mycotoxins, In: S. Natori, K. Hashimoto, Y.Ueno (Editors). Mycotoxins and phycotoxins. Elsevier, Amsterdam, pp. 69-80

Josephy P.D., Lord H.L., Snieckus V.A., 1990. Inhibition of benzo[a]pyrene dihydrodiol epoxide mutagenicity by synthetic analogues of ellagic acid. Mutat. Res. 242, 143-149

Kado N.Y, Langley D., Eisenstadt E., 1983. A simple modification of the Salmonella liquid incubation assay. Increased sensitivity for detecting mutagens in human urine. Mutat. Res. 121, 25-32

Kuo M., Lee K., Lin J., 1992. Genotoxicities of nitropyrenes and their modulation by apigenin, tannic acid and indole-3-carbinol in Salmonella and CHO systems. Mutat. Res. 270. 87-95

Lesca P., 1983. Protective effects of ellagic acid and other plant phenols on benzo[a]pyrene-induced neoplasia in mice. Carcinogenesis 4 , I $65 \mathrm{I}-1653$

Liu L., Massey T.E., 1992. Bioactivation of aflatoxin B1 by lipoxygeneses, prostoglandin H synthase and cytochrome P450 monooxygenase in guinea-pig tissues. Carcinogenesis 13, 533-539

Lord H.L., Snieckus V.A., Josephy P.D., 1989. Re-evaluation of the effect of ellagic acid on dimethylnitrosamine mutagenicity. Mutagenesis 4, 453-455

Rossi M., Erlebacher J., Zacharias D.E., Carrell H.L., lannucci B., 1991. The crystal and molecular structure of ellagic acid dihydrate: a dietary anti-cancer agent. Carcinogenesis 12, 2227-2232

Song P.S., Tapley K.J., 1979. Photochemistry and photobiology of psoralens. Photochem. Photobiol. 29, 1177-1197

Stavric B., 1994. Antimutagens and anticarcinogens in foods. Food Chem.Toxic. 32, 79-90

Stich H.F., Powrie W.D., 1984. Naturally occurring phenolics as antimutagenic and anticancerogenic agents. In: M. Friedman (Editor). Nutritional and toxicological aspects of food safety. Plenum Press, New York, pp. 1-29

Wattenberg L.W., Leong J.L., 1970. Inhibition of carcinogenic action of benzo[a]pyrene by flavones. Cancer Res. 30, 1922-1925

Wattenberg L.W., Coccia J.B., Lam L.K.J., 1980. Inhibitory effects of phenolic compounds on benzo[a]pyrene induced neoplasia. Cancer Res. 40, 2820-2823 